NOTICIAS Y COMENTARIOS 


\title{
LA INFORMACIÓN GEOGRÁFICA EN INTRARREDES CORPORATIVAS. APLICACIÓN DE UN SIG PARA LA GESTIÓN DEL CAMPUS DE LA UNIVERSIDAD DE ALICANTE: SIG-UA
}

\author{
Alfredo Ramón Morte* \\ Ángel Sánchez Pardo \\ José M. Mira Martínez
}

\section{RESUMEN}

SIG-UA es un proyecto de investigación de Geografía aplicada destinado a la elaboración y puesta en explotación de un sistema de información geográfica (SIG) para la gestión de espacios e infraestructuras de la Universidad de Alicante. El objetivo es demostrar cómo la contemplación gráfica de la información contenida en bases de datos, es decir, su representación cartográfica, es el medio más eficaz para comprender y utilizar estrategias de actuación territorial, ventajas que se ven potenciadas al ser usadas junto a otra forma muy sugerente de consulta de datos, los protocolos de transferencia de hipertextos en una red local de tipo corporativo. En apretada síntesis, los servidores WEB de aplicaciones SIG pueden estar llamados a convertirse en una extraordinaria opción para el control adecuado de los activos inmuebles, tanto en empresas privadas como en organismos públicos.

Palabras clave: SIG, WEB, intrarredes, gestión de espacios e infraestructuras.

\begin{abstract}
SIG-UA is a research project on applied Geography intended for the elaboration and exploitation of a geographic information system (GIS) for the spaces and infraestructures management of the University of Alicante (Spain). The main aim is to show how the graphic contemplation of the information which is kept in database, that is, its cartographic representation, it is the most effective way to understand and make use of territorial action strategies. These advantages are improved when they are used together with another interesting way of database consulting, namely, the HTTP in a intranet with TCP-IP protocol. To sum up, in order to control the property assets in a suitable way, both in private enterprises, state bodies and organs of the government, the WEB servers of GIS applicationes may become an oustanding choice.
\end{abstract}

Key words: GIS, WEB, intranets, spaces and infraestructures management.

* E-Mail: alfredo.ramon@ua.es 


\section{Los sistemas de información y la cartografía actual}

Son principalmente dos las cuestiones que caracterizan la reciente evolución de la Cartografía: la importancia e interés que viene despertando en diversos ámbitos de la sociedad actual y la evolución dinámica de las técnicas utilizadas en las diferentes fases del proceso de producción de mapas ${ }^{1}$.

Mediante las nuevas formas de cartografía se ejerce un dominio más efectivo sobre un espacio cada vez más complejo, con enormes cantidades de variables activas. La eficacia de la nueva Cartografía es tal que permite considerar, además de los beneficios potenciales de nuestros actos, las consecuencias de los mismos y los riesgos que implican. De esta manera, la Cartografía de la «Era de la Información» no lo es por realizarse con medios informáticos, sino por su intención de responder a estas preocupaciones sociales y ambientales de mayor alcance ${ }^{2}$.

La nueva cartografía temática es dinámica y multitemporal, pero sobre todo, interactiva. Facilita el control de información voluminosa y difícil, para aportar los resultados de una manera más eficaz, pues el propio usuario se puede convertir en el productor del mapa temático digital, gracias a una serie de recursos técnicos que le permiten trabajar de manera directa con el «dominio de la información» geográfica.

Los centros públicos y las empresas especializadas cada vez se preocupan menos por la producción de mapas impresos; el reto consiste en centralizar, organizar, actualizar, normalizar y controlar la existencia de grandes bases de datos geográficas para conseguir que lleguen a los lugares de trabajo, investigación y de decisión política de la forma más fácil y adecuada, en forma de resumen estadístico o en una cartografía casi hecha a la medida de las necesidades del que la consulta. La Cartografía automatizada es, por lo tanto, el reflejo funcional de las necesidades de la Sociedad de la Información, al convertirse en una interfase modélico-gráfica muy eficaz en los DSS (Decision Support System, Sistemas Soporte de toma de Decisiones), dentro de los MIS (Management Information Systems, Sistemas de Información para la Gestión y el Planeamiento) ${ }^{3}$.

La consulta de datos estratégicos sobre el territorio, a través de una modelización espacial de su ubicación sobre la superficie terrestre, está ofreciendo una vía de trabajo de gran fecundidad en las cuestiones relativas a la ordenación del territorio. La intervención de carácter interdisciplinar, propia de las tareas de planificación, encuentra en la moderna Cartografía un lenguaje gráfico que facilita la interpretación y uso de la información aportada por diversos grupos profesionales, a la par que amplia su capacidad temática funcional.

Desde el punto de vista científico, el proyecto que aquí se expone está facilitando la investigación de las posibilidades que ofrece esta vertiente cartográfica reciente, al poner a prueba las prestaciones de los sistemas de información geográfica, dentro de los MIS y

1 SANCHO COMINS J.; BOSQUE SENDRA, J. y CHUVIECO SALINERO E. (1996): «La Cartografía, los SIG y la teledetección en la reciente evolución de la Geografía española». La Nueva Realidad Geográfica en España. Aportación de la A.G.E. al $28^{\circ}$ Congreso Internacional de Geografía La Haya, 1996. En el Boletín de la A.G.E. no $21-22$, p. 151.

2 RAMÓN MORTE, Alfredo (1997): Sistemas de Información Geográfica en la Actividad Turística, en el curso Nuevas Tecnologías aplicadas al Turismo. Universidad Internacional Menéndez Pelayo (Valencia).

3 La Cartografía automatizada debe mucho de su razón de ser a los Sistemas de Información Geográfica y éstos, a su vez, a los sistemas más generales, basados en la gestión informática de grandes volúmenes de datos para la toma de decisiones (SCOTT MORTON, M.S. (1971): Management Decision Systems: Computer Based Support for Decisión Making. División de Investigación. Universidad de Harvard, Cambridge. Massachusetts. Y Densham, P.J. (1991): Spatial Decision Support Systems. En Maguire, D.; Rhind, D. y Goodchild, M. (eds.) Geographical Information Systems: Principles and Applications, Londres. Longman, pp. 403-412. 
$D S S$, en la gestión de información contenida en bases de datos y su relación con la ubicación espacial de los fenómenos. Además, permite evaluar la idoneidad de la cartografía digital, automatizada o «inteligente» desde las últimas tendencias de producción y explotación dentro de esta disciplina instrumental, actualmente marcada por un fuerte sesgo tecnológico de tipo informático ${ }^{4}$.

En este sentido, surgen cada vez más aportaciones científicas que tratan de dar una dimensión aplicada a todas estas cuestiones, utilizando los recursos que aquí se exponen, es decir, mediante la utilización en una intranet (intrarred) o red local corporativa que hace uso de las herramientas informáticas propias de Internet ${ }^{5}$. De hecho, a muy corto plazo, estos sistemas basados en protocolos de transferencia de hipertextos, por su capacidad gráfica para la consulta y actualización de bases de datos, pueden convertirse en el medio más eficaz de explotación de los SIG.

Por tanto, constituye una línea de investigación y desarrollo que se integra en la utilización combinada de SIG, bases de datos, hipertextos, recursos multimedia e hipermedia, para evaluar las posibilidades que ofrece la sinergia derivada de la coordinación en tiempo real de equipos técnicos y grupos humanos dispersos geográficamente, ya sean de la propia organización, como en el caso que se presenta, o del mercado al que van dirigidos los servicios y productos; cuestiones, todas ellas, que hace pocos años constituían auténtica ciencia ficción en nuestro país.

\section{Las necesidades de un campus universitario del siglo XXI}

Atender a una «población» aproximada de cuarenta mil personas entre alumnos, personal laboral y profesores, obliga a la elaboración de una herramienta efectiva para la adopción de criterios de actuación y planificación de la dotación y uso de equipos e infraestructuras en un campus universitario. Las aplicaciones informáticas que ya existían en muchos de los negociados y órganos de gobierno de la Universidad de Alicante presentaban el inconveniente de una gran heterogeneidad e independencia en su organización, con las dificultades consiguientes de aprovechamiento y actualización de unos datos caracterizados por su naturaleza dinámica, condigna al crecimiento experimentado recientemente por esta institución, con constantes modificaciones internas de los edificios, construcciones de nueva planta, cambios en las actividades, traslados e incorporaciones de personal.

Por estas razones, se comenzó la elaboración de $S I G-U A$ en el Laboratorio de Cartografía Automatizada y SsIG del Instituto Universitario de Geografía, configurando un sistema destinado a la gestión de este campus, con el que dar oportuna respuesta, de forma fácil e interactiva, a las necesidades de información y organización de las bases de datos de Gestión Económica, Gestión de Espacios y Servicio de Personal, bajo una doble premisa, por un lado, la utilización de cartografía «inteligente» como intermediario gráfico más eficaz en la consulta y tratamiento de bases de datos estratégicas para la resolución de problemas espaciales, por otro, la mejora y facilidad para su explotación operativa por parte de los usuarios, gracias a su puesta en servicio a través de una Intrarred (Intranet) corporativa, centralizada en un Servidor $W E B$ y surtida por una base de datos distribuida entre los diferentes órganos de gestión, mediante la red local institucional de la universidad.

4 RAMÓN MORTE, Alfredo (1997): Op. cit.

5 NUNES, J.; FERRERO, I.; MANZANO, A. E. ITURBE, A.: «SIG-CAMPUS: Una aplicación de SIG corporativo integrada en los servicios Internet/Intranet de una organización compleja» en la III Semana Geomática de Barcelona. Colegio Oficial de Ingenieros Técnicos de Topografía de Cataluña e Instituto Cartográfico de Cataluña. Barcelona, abril de 1997. 
La referenciación geográfica de todos los inmuebles, instalaciones y estancias del campus permite que las relaciones geométricas de localización, extensión, dimensión, vecindad, proximidad, acceso o superposición, entre otras, conviertan al espacio geográfico en el elemento vertebrador de las diferentes bases de datos, que por su temática eran de gran complejidad y variación (personal, gestión económica, asignación de espacios, oficina técnica...). La representación geográfica digital, mediante el nexo que un SIG es capaz de establecer con datos alfanuméricos, se convierte en «inteligente» y enriquece cualquier sistema de información basado en tablas relacionales.

En efecto, una universidad es una buena «parcela de experimentación» para ello, pues cada aula, cada laboratorio, cada seminario o despacho es escenario de actividades muy diversas y requieren de una dotación de servicios muy específicos y variados en extremo, según la disciplina científica o incluso el área de conocimiento que hace uso de ellos; mención aparte son los espacios comunes, de ocio, circulación, servicios generales y un extenso número de variables informativas a considerar. Sin embargo, todos comparten el hecho de ocupar un espacio geográfico muy concreto, lo que permite ponerlos en relación entre sí, máxime cuando se trata de establecer una lógica para la asignación de estancias, la dotación de servicios especializados y la previsión del crecimiento de la universidad.

Con este ánimo se ha diseñado el SIG de la Universidad de Alicante, SIG-UA, basado en la digitalización de los planos y la cartografía del campus y su conexión a las bases de datos de información estratégica, con la integración de tres módulos principales, perfectamente comunicados entre sí: SIGEP (sistema de información para la gestión económica y patrimonial), SIPER (sistema de información para la gestión de personal) y SIGES (sistema de información para la gestión de espacios, equipos y servicios), todos ellos pensados para su explotación a través de una intranet corporativa, que trabajaría contra las tablas asociadas a datos geográficos, de espacios, personal y servicios económicos, almacenadas y gestionadas desde un ordenador con programas como ARC/INFO, ARCVIEW GIS, MGE de Intergraph y AUTOCAD MAP, para realizar su divulgación mediante un Servidor WEB con el programa GEOMEDIA WEB MAP, capaz de leer e integrar en un sólo proyecto diferentes formatos de SIG, generando un método simple de explotación de la información basado en la utilización de navegadores de internet, a través de entornos gráficos combinados y complementarios de hipertexto y cartografía vectorial en formato CGM o raster.

La experiencia está resultando enriquecedora para la explotación corporativa funcional y efectiva de sistemas de información geográfica, tanto por la dificultad que ha supuesto la complejidad de unas instalaciones universitarias, como ya se ha comentado, como por su potencial aplicación a otro tipo de centros públicos, como ayuntamientos, direcciones generales (ordenación del territorio, planeamiento urbano, medio ambiente o protección civil), diputaciones provinciales y empresas privadas (como grandes superficies comerciales, asociaciones de pequeños comerciantes de centros urbanos o agencias de la propiedad inmobiliaria); es decir, cualquier grupo profesional cuya actividad se oriente a la dotación de servicios especializados relacionados con una vertiente de actuación espacial o patrimonial.

Teniendo en cuenta la utilidad potencial de estos sistemas y dado que la Universidad de Alicante ha experimentado un crecimiento sobresaliente a lo largo de los últimos años, con un campus extenso y complejo, fruto de un alto número de matriculados y de la variada oferta de titulaciones, se trataría de mantener y aumentar, si cabe, la calidad en la enseñanza, que pasa por controlar y planificar al máximo la dotación de espacios y servicios, constituyendo estas cuestiones clave importante de la estrategia del equipo de gobierno.

El objetivo principal de esta propuesta sería, proporcionar a los servicios gestores y de organización recursos tecnológicos que permitan conocer con alto grado de detalle la 
distribución de espacios y servicios, así como la inclusión de herramientas informáticas capaces de ofrecer soluciones a los diferentes problemas que sean planteados en el ejercicio de estas funciones.

Un sistema de estas características permite:

1. Conocer la proporción, distribución y uso de superficie por cada unidad de gasto, según los diferentes temas a considerar (investigación, docencia, administración...).

2. Racionalizar la asignación actual de espacios y servicios.

3. Pronosticar, mediante la elaboración de modelos prospectivos, la demanda ocasionada por el crecimiento de la universidad.

4 Planificar, con meticulosidad, las necesidades y el uso futuro de las infraestructuras universitarias.

5. Proporcionar amplias prestaciones con el mínimo esfuerzo de reciclaje profesional y aprendizaje por parte del personal técnico y administrativo.

6. Permitir que los usuarios responsables de cada nivel temático puedan ampliar o modificar la información del sistema, ayudados por la referencia gráfica e interactiva (CGI de Internet) de los mapas digitales. Estableciendo para ello los correspondientes protocolos de acceso y seguridad.

En este sentido, un SIG puede funcionar como un modelo virtual a escala real de la Universidad de Alicante, sin olvidar su nexo de unión a las correspondientes tablas relacionales de carácter temático. Esta organización lógica habilita al usuario y al gestor o analista para actualizar datos, consultarlos e incluso plantear supuestos con una doble vía de acceso, es decir, mediante operaciones visuales, partiendo de los planos digitales, o desde las propias bases de datos que existen en los diferentes servicios universitarios, obteniendo una rápida referencia visual del inmueble al que hace referencia la información con la que se trabaja. Todo esto, sin entrar a comentar los beneficios inducidos que representa, para la Oficina de Planificación y Proyectos y para el propio Vicerrectorado de Infraestructuras, disponer de la cartografía y los planos detallados de todo el campus en formato digital.

Una de las principales ventajas del empleo de estos sistemas respecto al uso de bases de datos normales, es la capacidad de realizar un análisis espacial complejo de la información, donde los datos adquieren siempre situación, dimensión y volumen, incorporando a las operaciones relacionales habituales el correspondiente mapa o plano de las estancias, instalaciones o edificios implicados en el proceso, así como la posibilidad de acceder, a través de esta representación gráfica, a los atributos relativos a sus usuarios o equipos, configurando una aplicación informática para ejercer un control total sobre la organización de una demarcación territorial concreta. Es así como el espacio geográfico se convierte en un elemento clave dentro del modelo entidad-relación de las bases de datos.

Unos supuestos pueden ilustrar de manera más eficaz lo hasta ahora expuesto; así, un usuario de SIG-UA, desde la red local de la Universidad de Alicante, con un simple navegador de internet, puede consultar una mapa del campus, en una página del WEB oficial de la universidad. Sólo con hacer uso del ratón, sobre un edificio concreto, accede a una conexión con otra página en la que le muestra los planos de sus diferentes plantas y estancias, llegados a este punto, mediante menús de contexto o botones de control, selecciona una de entre una variada gama de operaciones (consultar las dimensiones o la capacidad de alumnado de una habitación; el nombre del encargado, si se trata de un laboratorio; mandar un mensaje de correo electrónico al servicio de gestión de espacios para reservar dicha aula para un examen o notificar, al profesor de un despacho elegido, la 
petición de cita en horas de tutoría). Incluso con los permisos correspondientes y a través de formularios, se puede consultar información de las diferentes bases de datos conectadas a una estancia determinada, mientras la contemplamos en pantalla, o introducir o cambiar los registros ya existentes, gracias a un formulario simple del mismo documento de macrolenguaje de hipertexto, cuando se produce un cambio de actividad o traslado de personal.

Estas funciones, entre otras, pueden hacer pensar la entidad de los cambios en el proceso administrativo y la agilidad que pueden adquirir determinadas operaciones cotidianas de la vida universitaria. En el gráfico 1, en la parte superior se observa una pantalla de un visualizador típico de datos de un sistema de información geográfica (Arcview GIS $3.0 a$ ), en la que se aprecia la fácil realización de consultas gráficas y tabulares, mientras que la parte inferior corresponde a una ventana de aplicación en la que se ejecuta un navegador de internet con una página web dividida en marcos, dónde la consulta de datos y su visualización gráfica es todavía mucho más sencilla.

\section{Componentes y elementos del $S I G-U A$}

El conjunto de los recursos que se han puesto en funcionamiento para la elaboración del $S I G-U A$ constituye un referente de economía de medios y esfuerzos, encaminado a la obtención de la máxima eficacia a muy bajo coste. La diversidad de servicios y estamentos de un ámbito tan heterogéneo y multidisciplinar como es la Universidad de Alicante requiere, como ya se ha apuntado, racionalizar y homogeneizar la información existente en la misma. Con estas premisas se gestó en el Vicerrectorado de Infraestructuras y Servicios la creación de un Sistema de Información Integral que fuera capaz de resolver la gestión de estas cuestiones con agilidad.

La Universidad de Alicante dispuso para ello los medios necesarios para el desarrollo del proyecto, una vez asumida la financiación, tal y como se expresa en Tabla I adjunta, para ser adjudicada al Laboratorio de Cartografía Automatizada y SsIG del Instituto Universitario de Geografía la creación del sistema, dada la experiencia en la aplicación de estas herramientas informáticas en cuestiones de Geografía Aplicada ${ }^{6}$, contando para ello con la colaboración del profesorado del Departamento de Lenguajes y Sistemas Informáticos para los temas relativos a sistemas de redes entre ordenadores y desarrollos en $H T M L$, CGI y Java, propios del WEB.

En este sentido, en la fase de producción del SIG, la existencia de un equipo científico y técnico ya formado y especializado en estas cuestiones, además de la disposición de un equipamiento físico y lógico adecuados, con casi una década de experiencia y constante actualización, ha tenido un papel prioritario en la disminución de los costes económicos. Este aspecto relevante, además del abaratamiento, ha permitiendo reducir el plazo de ejecución a tan sólo doce meses.

6 Por hacer mención de algunos referentes, merece la pena destacar las aplicaciones de SS.I.G. realizadas por el I.U.G. de la Universidad de Alicante en diversos temas de ordenación o gestión del territorio: estudios de aptitud del medio físico como el realizado para el proyecto urbanístico del «Triángulo Alicante-Elche-Santa Pola, el estudio evolutivo del planeamiento urbanístico del municipio turístico de Torrevieja o evaluación de espacios de calidad ambiental dentro del proyecto «SIGMA», tal y como se refiere en la bibliografía que acompaña a esta ponencia. 


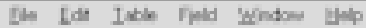

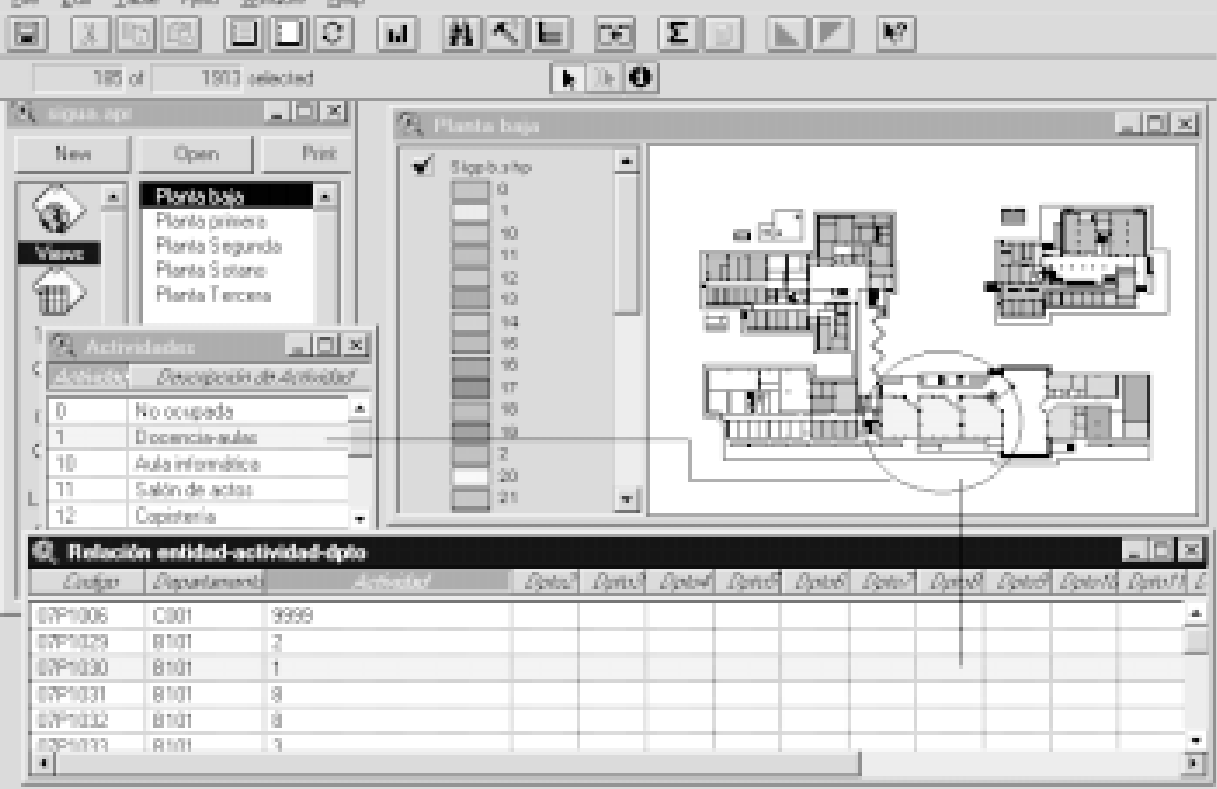

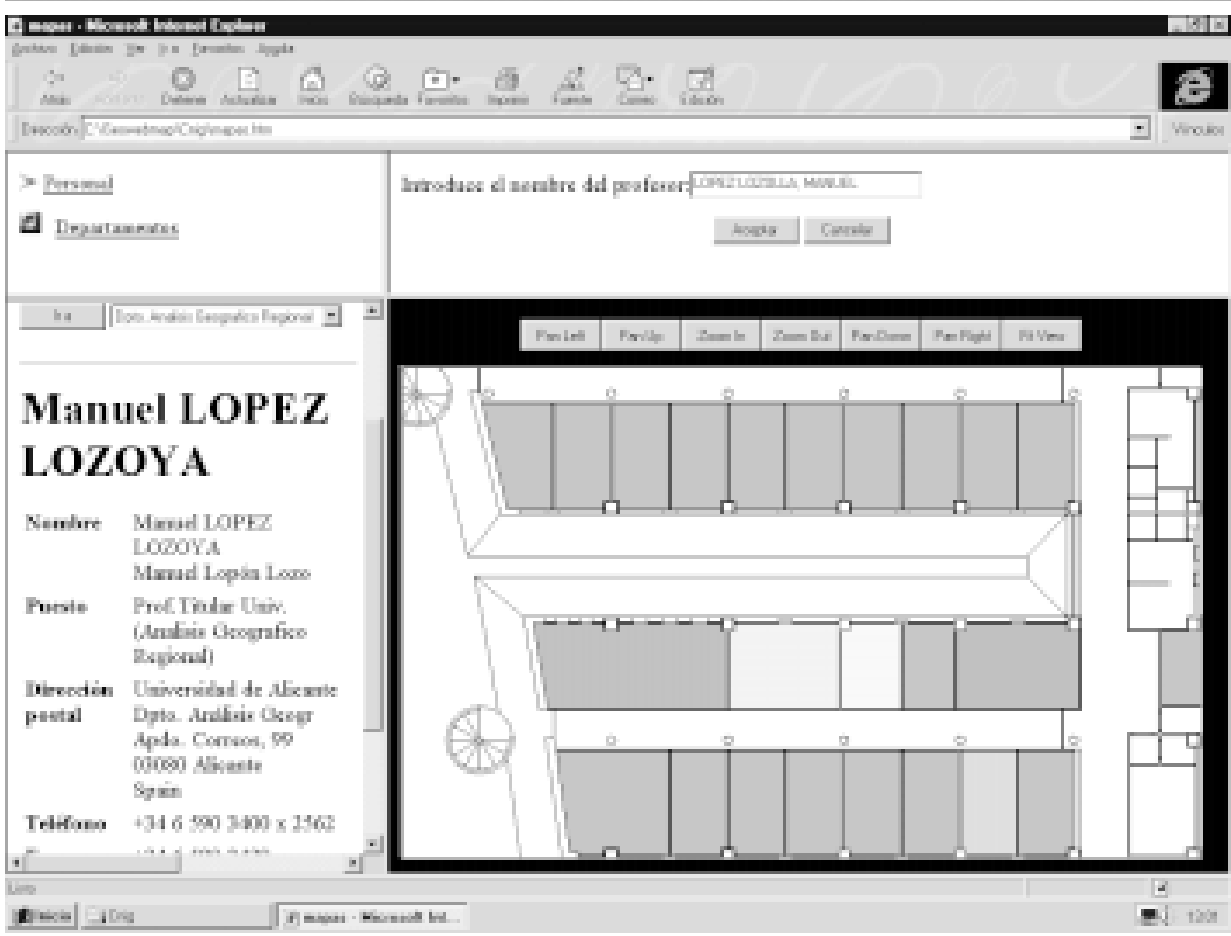

GRÁFICO 1 


\begin{tabular}{|l|r|}
\hline Gastos de producción & Importe (aprox.) \\
\hline Elementos Físicos & $1.095 .000 \mathrm{Pts}$ \\
Elementos Lógicos & $300.000 \mathrm{Pts}$ \\
Equipo Humano & $3.780 .000 \mathrm{Pts}$ \\
Otros & $300.000 \mathrm{Pts}$ \\
\hline Subtotal & $5.475 .000 \mathrm{Pts}$ \\
\hline Gastos de explotación & Intranet Corp. \\
\hline Elementos Físicos & $3.250 .000 \mathrm{Pts}$ \\
Elementos Lógicos & $1.400 .000 \mathrm{Pts}$ \\
Formación & $150.000 \mathrm{Pts}$ \\
Equipo Humano y Mantenimiento técnico (anual) & $2.000 .000 \mathrm{Pts}$ \\
Otros & $150.000 \mathrm{Pts}$ \\
\hline Subtotal & $6.950 .000 \mathrm{Pts}$ \\
\hline
\end{tabular}

\section{Fases de trabajo}

Para conseguir los objetivos expuestos con los medios a los que se ha hecho referencia, se establecieron las fases de trabajo a desarrollar y se programaron los plazos de ejecución sintetizados en los puntos siguientes:

I. Reunión con los interesados, recopilación y organización de la información (las reuniones se siguen celebrando de manera periódica mediante una comisión se seguimiento del proyecto).

II. Diseño del SIG (creación y organización del equipo humano, del equipo lógico, del equipo físico, determinación de costes, de la funcionalidad del sistema, de la calidad de la información, de las bases de datos, de las bases cartográficas y de los problemas de explotación y actualización).

III. Experiencia piloto en una zona restringida del campus.

IV. Ejecución de la parte principal del proyecto. Producción del SIG-UA.

V. Desarrollo de la fase de explotación (fase actual): diseño de las estrategias de explotación, definición de las funciones más importantes y usuales, economía de medios y esfuerzos en la actualización del sistema y la formación de personal usuario.

\section{Diseño del SIG-UA}

La importancia de esta fase de trabajo mediatiza de forma notable el desarrollo del proyecto, puesto que es aquí donde los organismos receptores establecen los criterios que debe cumplir el sistema, planteando sus necesidades, acordes con la información preliminar existente. En este sentido, se ha hecho especial hincapié en las vías de aprovisionamiento, la estructura y comprensión de las bases de datos, así como los medios para llevar 
a cabo los requisitos planteados, valorando, en su justa medida, las posibilidades técnicas de incorporarlas al sistema. Ésta, es una forma de evitar esfuerzos innecesarios en el transcurso del proyecto y ahorrar tiempo de ejecución.

Una vez planteadas las necesidades, el equipo técnico define las estrategias globales de desarrollo del SIG, centradas en resolver las siguientes cuestiones:

- Determinación, formación y reparto de responsabilidades del Equipo Humano.

- Perfilar las fuentes de información que proveerán al sistema, contactando con los órganos competentes y cursando las solicitudes pertinentes, encuestas y sus correspondientes trámites, incluidas las periódicas actualizaciones de información.

- Acondicionamiento del equipo físico, sus características y puesta a punto, según los dos puntos anteriores (en el Laboratorio de Cartografía Automatizada y SsIG, se disponía ya de un equipo para los fines propuestos ${ }^{7}$.

- Definición de las aplicaciones informáticas necesarias para cada fase, para lo cual se parte de la ventaja inicial expuesta en el punto anterior, aunque conviene matizar la incorporación de una estación para cumplir la función de Servidor WEB y el software necesario para la explotación y actualización de $S I G-U A$ a través de una Intranet Corporativa de la Universidad de Alicante, por considerar que esta estrategia puede suponer el éxito del sistema en la última fase (explotación del SIG), al simplificar la formación del equipo humano destinado a su uso, las correspondientes tareas de actualizar la información y abaratar la inversión en equipos lógicos y físicos que sería necesaria con otros planteamientos de red local.

El acceso a fuentes de información y su utilización suele presentar el problema añadido de hacer rentable una variada gama de formatos y diseños de procedencia externa al equipo de trabajo y de gran heterogeneidad, dando solución a problemas de incompatibilidad entre ficheros, desigual calidad, diferencias en la escala de trabajo, en los objetivos de su creación e incluso de la integridad de los datos.

Respecto a estos problemas en los controles de calidad de información, es preciso mencionar la ventaja comparativa que supone desarrollar un proyecto para la Universidad de Alicante desde un órgano interno, al disponer de datos actualizados y veraces, a partir de las bases de datos existentes en el Centro de Proceso de Datos de la Universidad de Alicante, con formatos traducidos a DBASE o ACCESS, para finalmente ser gestionados en SQL SERVER.

Por otra parte, es de agradecer la inestimable colaboración de la Oficina Técnica de Planificación y Proyectos de la Universidad de Alicante, que puso a disposición del equipo de desarrollo los planos de las plantas de todos los edificios y centros universitarios, en la mayor parte de los casos con un formato digital de tipo vectorial, ahorrando un gran trabajo de digitalización, para poder aumentar el esfuerzo dedicado a las labores de corrección de errores y adecuación de los formatos gráficos, para su inclusión en un SIG, dada la exigencia en la correcta disposición de la geometría y la exactitud de las referencias geográficas en un sistema de coordenadas planas.

7 El I.U. de Geografía cuenta con una estación de trabajo UNIX con ARC/INFO y una estación gráfica para Windows-NT con los módulos de SIG de INTERGRAPH, conectadas a una red local de siete PCs, en los que se dispone de las correspondientes licencias de ARCVIEW, ARCCAD, MGE, Microstation, GEOMEDIA y Autocad MAP, además de programas de diseño gráfico y dibujo. Todo ello, contando con la ventaja del trabajo en un equipo integrado que hace uso de una amplia gama de periféricos: mesas digitalizadoras, scanners, plotters e impresoras de calidad, elementos de seguridad y soportes de almacenamiento masivo, con acceso a la red TokenRing institucional de la Universidad de Alicante. 
Las aplicaciones informáticas y equipos utilizados en $S I G$-UA pueden clasificarse en dos grandes grupos, dependiendo de su utilización, según se trate de la fase de producción o elaboración del sistema, o de la fase de utilización y explotación por parte de los usuarios.

\section{La producción del sistema de información}

Una vez determinados los datos necesarios, la posibilidad de su incorporación al sistema y las funciones útiles que debían cumplir, se procedió a su organización y a la construcción del modelo entidad-relación, procurando una estructura versátil y abierta, para facilitar la perfecta integración del grueso de bases de datos que se tomaban de partida. De esta manera, a muy grandes rasgos, se inició el trabajo sobre la base de la existencia de dos niveles de información claramente diferenciados:

A. Información geográfica o geométrica: organizada en niveles espaciales o capas de información, en el que cada cobertura espacial hace referencia a las plantas tridimensionales de la Universidad de Alicante, desde los sotanos hasta las azoteas de todos los edificios existentes ${ }^{8}$. Todos las variables geográficas bidimensionales (puntos, líneas o áreas) fueron etiquetadas bajo un riguroso criterio de clasificación, desde los elementos más singulares (conducciones, muros exteriores, tabiques, aseos, huecos de ascensor, accesos, etc.) hasta llegar a las estancias que disponen de un modelo de clasificación similar al empleado en los índices bibliográficos.

B. Atributos temáticos o alfanuméricos: organizados en tablas relacionales suministradas por los centros de gestión (como ya ha sido indicado) y exportadas a formatos para ser gestionadas por $S Q L$ Server, como medio de integración en el sistema.

Una vez completada esta fase fue necesario enlazar ambos grupos de información a través de campos indexados, para la unión entre los atributos geográficos y los alfanuméricos, adecuando el modelo entidad-relación a una clave principal, correspondiente al campo que albergaba el código de identificación ${ }^{9}$ de cada una de las variables geográficas que definen los activos inmuebles (de forma principal, cada uno de los habitáculos que aparecen en las diferentes plantas de los edificios del campus).

Cada una de estas entidades geográficas dispone, pues, de una identificación única que proporciona una reseña escueta de cada unidad de actuación, presente en la tabla de datos geográfica o geométrica (capa gráfica o mapa digital) como un campo más que acompaña a los identificadores internos generados por los programas de SIG utilizados. Así, a partir de este campo clave, ambos grupos de tablas de datos, las geométricas y las alfanuméricas, establecen un vínculos activo que integra los datos de personal, gestión de espacios y gestión económica, en los diferentes mapas de la universidad. Tal y como aparece reflejado en el esquema del modelo entidad-relación del SIG-UA que aparece a continuación, incluyendo los subsistemas de los proyectos de gestión SIGES (sistema de información

8 La Oficina de Transferencia de Proyectos suministró las plantas de cada edifico organizadas en ficheros con formato de intercambio nativo del paquete de diseño AutoCAD. Para facilitar la labor de inserción de plantas en cada nivel de información, se trató individualmente cada una de ellas, generando una cobertura individual que permitió corregir errores topológicos, transformarlos (cambios de escala y georreferenciación) y realizar trabajos de campo encaminados a comprobar la fiabilidad de los datos.

9 Esta codificación fue basada en el modelo de tesauro: los dos primeros dígitos hacen referencia al número del edificio, los dos siguientes a la planta (incluyendo los sótanos) y los tres últimos corresponden a un número secuencial y aleatorio empezando a partir del $\mathrm{n}^{\mathrm{o}} 001$. 


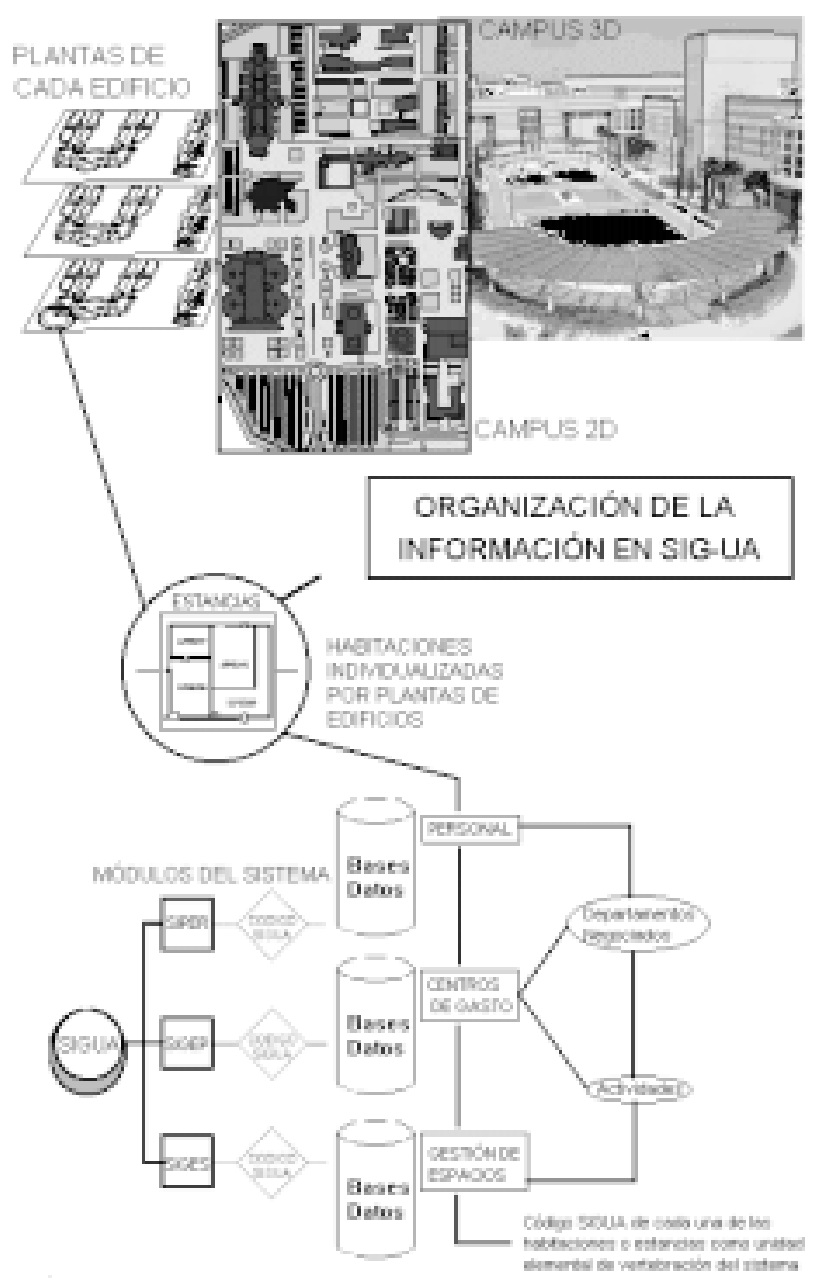

GRÁFICO 2

para la gestión de equipos, espacios y servicios), SIPER (sistema de información para la gestión de personal) y SIGEP (sistema de información para la gestión económica y patrimonial).

El uso de este variado modelo relacional, pese a la «heterodoxia» que supone la vertiente gráfica y geométrica, implica un aumento exponencial del potencial de un Sistema de Información o MIS. Sin embargo, todavía es muy frecuente encontrar organizaciones basadas en tablas relacionales simples, independientes e inconexas, entre las secciones de un organismo público o empresa. En el gráfico 2 se observa la organización de la información en el sistema y cómo el código específico y la representación gráfica de los activos inmuebles son los elementos clave de la relación entre las bases de datos de personal, centros de gasto y gestión de espacios, dando lugar los subsistemas ya citados.

Ante esta situación, la vertiente del análisis espacial y la posibilidad de incrementar la riqueza de información con salidas gráficas de mapas, hace de los SsIG una herramienta 
ideal para la gestión de la información por enriquecer las relaciones y servir de «intermediario» gráfico de consultas e informes.

Pero no todo son ventajas, pues un costoso equipo físico y lógico, las necesidades de actualización y control de calidad de los datos y las cuestiones problemáticas de formación de usuarios dentro del personal administrativo de la universidad, imponían una meditada estrategia de explotación, considerando ésta la clave para el éxito del SIG-UA. Al ser el punto de inflexión en el que comienza el fracaso de muchos sistemas de información que tuvieron una primera ejecución impecable, pero una nula previsión de su funcionamiento posterior de forma práctica y aplicada (caer en desuso por el coste de su actualización y la dificultad de su uso, antes de poder hacer patentes las ventajas inherentes a ello).

Atendiendo a estas consideraciones, en la fase de producción, ya concluida, ha intervenido un equipo humano, físico y lógico caracterizado por una alta especialización, elementos complejos y de alto precio (estaciones de trabajo gráfico, periféricos sofisticados y una extensa grama de programas comerciales como ARCINFO, ARCAD, ARCVIEW, MGE, MICROSTATION, GEOMEDIA, RIS Microsoft Data Server o SQL Server), cuyo principal inconveniente no es sólo su coste. En efecto, estos elementos requieren de la existencia de una formación técnica y experiencia en la elaboración de $S \mathrm{SIG}^{10}$.

Pero no se debe obviar que los usuarios finales, técnicos y personal administrativo, pueden carecer de la formación y la destreza necesaria en el uso de los medios citados, o del tiempo y esfuerzo necesarios para adquirirla, no proporcionados, en primera instancia, a los beneficios que puedan obtener como usuarios. Todo esto, sin contar con el nada despreciable coste de adquirir ordenadores y programas para tales fines en una red local con determinados protocolos.

\section{El sistema de explotación como clave del éxito del SIG-UA}

Partiendo de estas consideraciones, el equipo humano encargado de la fase de producción del $S I G-U A$, consideró que la viabilidad de su explotación partía de buscar un método simple, barato, que sin esfuerzo se ofreciese a un alto número de usuarios, con los sistemas de acceso y seguridad correspondientes a la protección de la integridad de los datos.

La utilización de información geográfica a través de una intranet corporativa se perfiló muy pronto como una innovación tecnológica capaz de dar una solución de contundencia (son muchos los SsIG que se crean, pero muy pocos los que de verdad se usan). Un servidor dedicado podía dar un rendimiento adecuado a bastantes usuarios de una red local $(L A N)$ y permitir, incluso, ofrecer la información con un protocolo TCP-IP (Internet) con un coste reducido. Para ello sólo ha sido necesaria la inversión en un ordenador debidamente equipado ${ }^{11}$ recurriendo a programas comerciales para ofrecer información geográfica a través de internet, como Geomedia Web Map, con la posibilidad de editar formularios de consulta, para ofrecer tablas dinámicas y actualizar la información alfanumérica y cartográfica mediante la ejecución de programas a tráves de la red (CGI o Pearl, entre otros), de entornos de trabajo editados en HTML y divididos en marcos.

10 FRANK, U. et al.: «Perspectiva de la tecnología SIG en los noventa» en Microstation Magazine, $\mathrm{n}^{\circ} 8$. Barcelona, 1994. 7-8 pp.

11 Un servidor WEB dotado de varios procesadores de 200 a $256 \mathrm{MHz}$., con $128 \mathrm{MB}$ de memoria RAM y $4 \mathrm{~Gb}$ de disco duro. Sistema operativo Windows NT 4.0, conectado a una estación de trabajo con un sistema gestor de bases de datos relacionales (SQL Server) y haciendo uso de las herramientas comerciales para SIG corporativo como GEOMEDIA WEB MAP. 


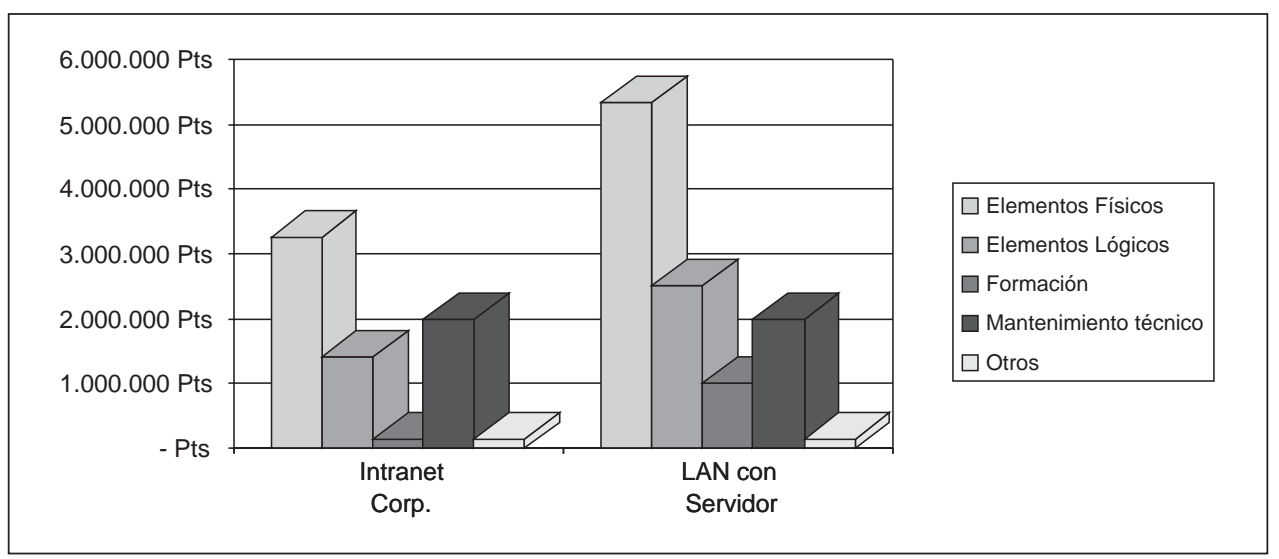

GRÁFICO 3. Gastos de explotación de SIG-UA

Esta opción, compleja para los encargados del desarrollo del proyecto, permitiría a los usuarios del SIG-UA tener sólo la responsabilidad de actualizar y consultar los datos mediante protocolos de macrolenguaje de hipertextos muy fáciles de utilizar, con un simple ordenador conectado a la red local de la Universidad de Alicante (ya dotada con su correpondiente página $W E B$ institucional y el necesario «cortafuegos»), a través de cualquiera de las «más que asequibles» versiones de navegadores de Internet, con las que la mayor parte del personal administrativo está ya familiarizados, sin necesitar conocimientos técnicos especiales para su uso.

Si se observa el gráfico 3, se pueden comparar las inversiones necesarias en pesetas de 1997 para la explotación del SIG-UA a través de una Intranet corporativa con protocolo TCP-IP o mediante una red local con servidor y diferente protocolo de comunicación, con un monto superior para casi todos los capítulos de gasto del último caso, destacando las inversiones en elementos físicos, lógicos y la formación de usuarios. Además, hay cuestiones que no son cuantificables desde el punto de vista del coste en pesetas simple. En efecto, el esfuerzo socio-profesional que puede suponer la formación y el reciclaje del personal en una empresa constituyen un serio inconveniente que puede dar lugar al fracaso de cualquier proyecto SIG, por muy bien que pueda estar el desarrollo realizado en su producción.

En el gráfico 4 aparece representado el esquema del sistema de explotación del SIG-UA, en el que se puede observar a modo de croquis las cuestiones antes comentadas de comunicación entre ordenadores y gestión de datos.

Todas estas razones son las que han motivado el sistema de explotación del SIG-UA, como un elemento principal a tener en cuenta en el diseño y elaboración del SIG, no albergando ninguna duda del enorme potencial de actuación de la cartografía «inteligente» para el uso de sistemas de información para gestión y planificación, a través de los hipertextos WEB de las intranets corporativas, es decir, un SIG corporativo.

\section{Los SsIG corporativos y la divulgación geográfica de datos}

El uso de sistemas de información geográfica convierte a la Cartografía digital o «inteligente» en un «intermediario» ideal para la consulta interactiva y dinámica de información de tipo estratégico, tanto para consultas e informes, como para actualizaciones. 


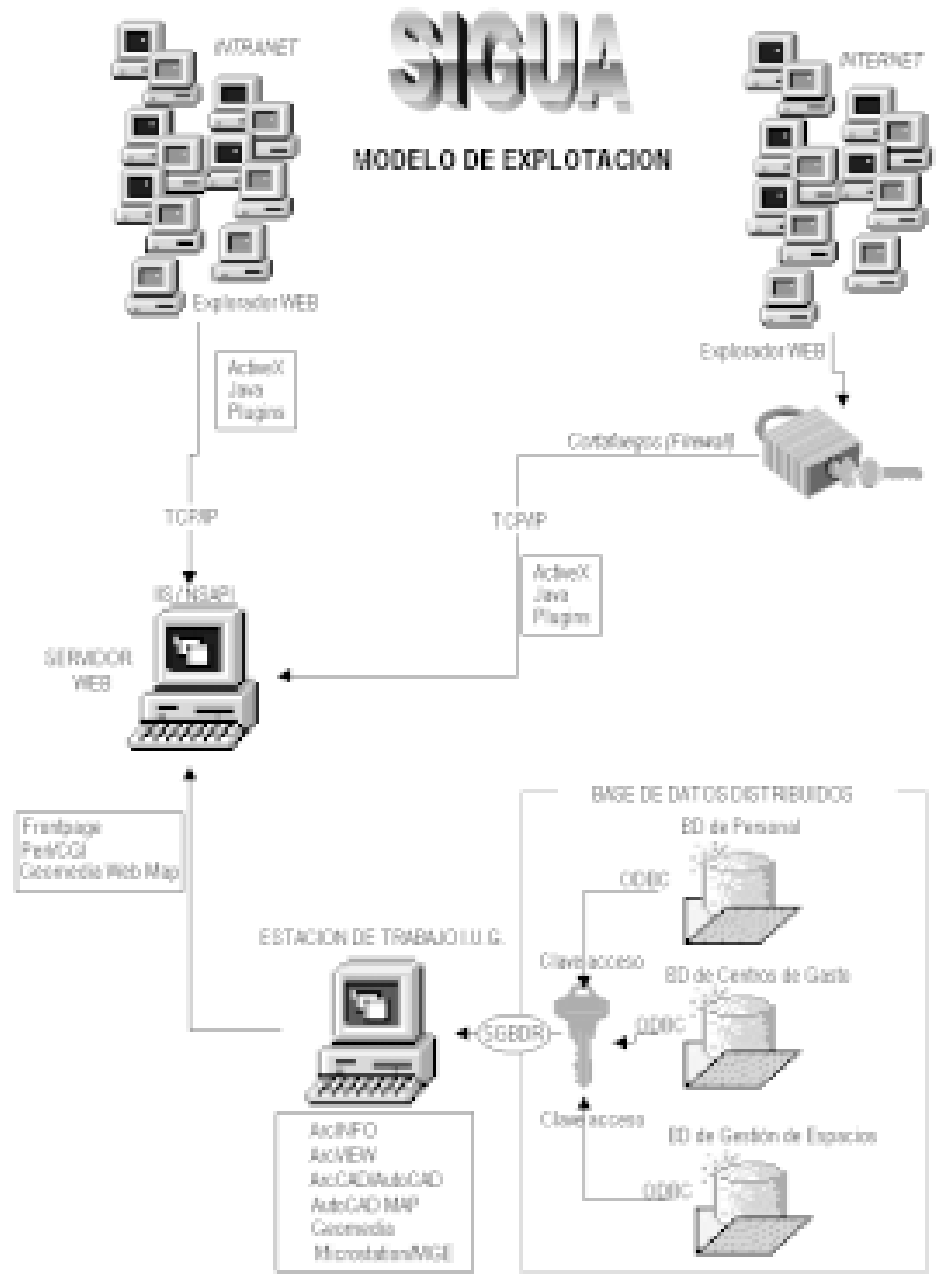

GRÁFICO 4

La versatilidad y eficacia de la consulta gráfica de información territorial ha revolucionado la propia disciplina cartográfica, que se ha visto enriquecida en sus vertientes básica y temática.

En la Universidad de Alicante ya está aportando ventajas de interés un sistema de este tipo, al permitir la edición de un Atlas de Espacios y Servicios con apéndices estadísticos que informan de la distribución de superficie por actividades, departamentos, centros de gasto y personal. Este tipo de información, sin duda, tendrá un valor estratégico a la hora de establecer un modelo de distribución de aulas, despachos, laboratorios, seminarios, entre otros tipos de estancias, que redundará en beneficio de toda la comunidad universitaria, en una sensible reducción de costes y en una mayor amortización de los activos inmuebles. 
Uno de los principales inconvenientes para su aplicación en organismos públicos es la inversión en equipo físico y lógico, pero mayor freno ha constituido la necesaria formación técnica para controlar la calidad y actualizar la información geográfica y alfanumérica de un SIG.

Conforme se va normalizando y centralizando la producción de este tipo de datos (que requiere un alto grado de especialización técnica) en los grandes centros públicos y determinadas empresas privadas o consultoras, el acceso se convierte en un problema de establecimiento de convenios de colaboración o de presupuesto económico, que deben ser proporcionados a la rentabilidad del uso del sistema.

Una traba mucho más infranqueable para su utilización generalizada, al margen de cuestiones económicas, es el gran esfuerzo de formación y reciclaje profesional que requiere el empleo de estos sistemas. En los últimos años, la existencia de potentes herramientas comerciales de visualización y consulta de Ss.I.G. (ARCVIEW o GEOMEDIA), incluso de desarrollos específicos (Map Objects o lenguajes de programación orientados a objetos), han permitido el acceso a esta tecnología por parte de profesionales liberales y cargos de la administración con un relativo grado de preparación.

No obstante, la posibilidad de hacer uso de los recursos potenciales de estas herramientas, a través de los entornos de hipertexto $W E B$, con las prestaciones de protocolos TCP-IP e intrarredes corporativas (intranets), amplía considerablemente el número de usuarios reales y de beneficiarios que, por otro lado, no necesitan ni equipos complejos, ni una preparación técnica esmerada (menor coste en dinero y esfuerzo sin menoscabo de las prestaciones).

Ante esta situación, es muy posible que la Cartografía «inteligente» producida por los SsIG y su explotación de forma corporativa en hipertextos, representen un campo de divulgación y desarrollo sin precedentes en la reciente, aunque intensa, historia de esta útil innovación tecnológica de tipo geográfico e informático.

$S I G-U A$, ha pretendido, por tanto, ser un proyecto con una doble finalidad: desarrollar una vía de investigación aplicada e innovadora, dentro del campo referido, sobre un caso complejo y difícil, para conseguir que los resultados tengan una aplicación práctica, útil e inmediata en la Universidad de Alicante y que sirvan como precedente de aplicación en otros organismos públicos y privados.

\section{Bibliografía}

AESIG: Anuario Geográfico 96. Asociación Española de Sistemas de Información Geográfica. Madrid, 1996.

BLOMAC, F. De: et al.: Arc/Info. Conceptes et applications en Géomatique. Ed Hermes. Paris, 1994.

CALVO MELERO, M.: «Necesidades actuales de divulgación y enseñanza de los Sistemas de Información Geográfica» en Ciudad y Territorio, no 89. Madrid, 1991.

DENSHAM, P.J. (1991): Spatial Decision Support Systems. En MAGUIRE, D.; RHIND, D. y GOODCHILD, M. (eds.): Geographical Information Systems: Principles and Applications, Londres. Longman, pp. 403-412.

FRANK, U. et al.: «Perspectiva de la tecnología SIG en los noventa» en Microstation Magazine, $\mathrm{n}^{\circ}$ 8. Barcelona, 1994.

GIS EUROPE. Europe's Geographic Information Systems magazine. Cambridge (1992-1996).

GOULD, M.: «El papel del geógrafo en el desarrollo de un SIG» en V Coloquio de Geografía Cuantitativa. Zaragoza, 1992.

GOULD, M.: «SIG en España . Perspectiva desde EE.UU.» en Boletín de la Asociación Española de Sistemas de Información Geográfica y Territorial , nº 2. AESIGYT. Madrid, 1992. 
MAPPING. Revista de Cartografía, Sistemas de Información Geográfica y Teledetección. Madrid, (1992-1996).

McABEE, J.L.: «La evolución de los SIG y la emergencia de soluciones puntuales para el usuario» en Microstation Magazine, $\mathrm{n}^{\circ}$ 8. Barcelona, 1994.

MIRA, J.M.; SÁNCHEZ, A. y RAMÓN, A. (1996): Un sistema de información geográfica para la gestión del medio físico-ecológico del sur de la Comunidad Valenciana en el libro: Cambios Regionales a finales del siglo XX (421-425 pp.). Universidad de Salamanca y Asociación de Geógrafos Españoles. 484 pp.

MOLDES, F.J.: Tecnología de los Sistemas de Información Geográfica. Madrid, 1995.

NUNES ALONSO, J.: «Evolución conceptual y técnica de los sistemas de información geográfica. La agenda pendiente», Boletín de la A.G.E., nº 9, 1989.

NUNES, J.; FERRERO, I.; MANZANO, A.E ITURBE, A.: «SIG-CAMPUS: Una aplicación de SIG corporativo integrada en los servicios Internet/Intranet de una organización compleja» en la III Semana Geomática de Barcelona. Colegio Oficial de Ingenieros Técnicos de Topografía de Cataluña e Instituto Cartográfico de Cataluña. Barcelona, abril de 1997. Inédito.

OCAÑA, C. et al.: «Nuevas técnicas en la investigación geográfica (Sistemas de Información Geográfica, Cartografía automática y teledetección)» en La Geografía en España (1970-1990). Real Sociedad Geográfica-Asociación de Geógrafos Españoles. Madrid, 1992.

RAMÓN MORTE, Alfredo (1997): Sistemas de Información Geográfica en la Actividad Turística, en el curso «Nuevas Tecnologías aplicadas al Turismo». Universidad Internacional Menéndez Pelayo (Valencia). EN PRENSA.

ROBINSON, A. et al.: Elements de Cartography. ( VI ${ }^{a}$ edición). Ed. John Wiley \& Sons, Inc. New York, 1995.

SÁNCHEZ, A., RAMÓN, A. y TORRES, F.J.: «Aplicación didáctica de PC Arc/Info al estudio ambiental del campo dunar de Maspalomas (Gran Canaria)» en $2^{\circ}$ Congreso de AESIG. Madrid, 1993.

SÁNCHEZ, A. y RAMÓN, A.: «Aplicación de un sistema de información geográfica en un estudio de capacidad de acogida del territorio (el Triángulo de Alicante, Elche y Santa Pola» Investigaciones Geográficas n 10, Alicante, 1992.

SANCHO COMINS, J.; BOSQUE SENDRA, J. y CHUVIECO SALINERO, E. (1996): «La Cartografía, los SIG y la teledetección en la reciente evolución de la Geografía española». La Nueva Realidad Geográfica en España. Aportación de la A.G.E. al $28^{\circ}$ Congreso Internacional de Geografía La Haya, 1996. En el Boletín de la A.G.E. no 21-22.

SCOTT MORTON, M.S. (1971): Management Decision Systems: Computer Based Support for Decisión Making. División de Investigación. Universidad de Harvard, Cambridge. Massachusetts.

TORRES, F.J. (1995): Aplicación de un S.I.G al estudio de un modelo de desarrollo local. Torrevieja 1956-1993). Instituto Universitario de Geografía de la Universidad de Alicante y el Excmo. Ayuntamiento de Torrevieja. 